\title{
UMA ANÁliSE PEDAGÓGICA DA ATUAÇÃO DA NEUROPSICOPEDAGOGIA FRENTE AO DESENVOLVIMENTO DAAPRENDIZAGEM E DA MEMÓRIA
}

\author{
Thiago Alves Miranda ${ }^{1}$
}

RESUMO: O tema do presente estudo é uma análise pedagógica da atuação da neuropsicopedagogia para o desenvolvimento da aprendizagem e da memória. Seu objetivo é analisar a importância da atuação da neuropsicopedagogia para o desenvolvimento do processo de aprendizagem, com foco no desenvolvimento dos sistemas de memória em sala de aula. Para o alcance desse objetivo, define e caracteriza o processo de aprendizagem considerando o caráter multifatorial de seu conceito revisa a relação entre a aprendizagem, o funcionamento executivo e a memória como complemento das funções executivas e destaca as contribuições da neuropsicopedagogia para a efetivação do processo de aprendizagem. A partir da realização de pesquisa bibliográfica qualitativa, conclui que a aprendizagem, o funcionamento executivo ou as funções executivas e a memória, processos psicológicos de ordem superior, não são isolados. Pelo contrário, a memória é o complemento das funções executivas e ambos permitem ao indivíduo gerar respostasadaptativas aos sistemas nos quais se encontra imerso. A aprendizagem necessita da memória para realizar os processos operativos e estratégicos na assimilação e nagestão do conhecimento, através da interação com a cultura e o meio. Tudo isso, a partir de uma postura integradora, destaca a importância da Neuropsicopedagogia para obter o conhecimento necessário sobre os construtos ou temáticas que dão origem e qualificam a aprendizagem humana.

Palavras-chave: Neuropsicopedagogia. Memória. Aprendizagem.

ABSTRACT: The theme of this study is the role of neuropsychopedagogy in the development and memory of learning. Its objective is to analyze the importance of the action of neuropsychopedagogy for the development of the learning process, focusing on the development of memory systems. To achieve this goal, it defines and characterizes the learning process considering the multifactorial character of its concept, reviews the relationship between learning, executive functioning and memory as a complement to executive functions and highlights the contributions of neuropsychopedagogy to the learning process effectuation.

Based on qualitative bibliographic research, it concludes that learning, executive functioning or executive functions and memory, psychological processes of a higher order, are not isolated. On the contrary, memory is the complement of executive functions and both allow the individual to generate adaptive responses to the systemsin which it is

\footnotetext{
${ }^{\text {I }}$ Doutorando em Direito Público e Evolução Social pela Universidade Estácio de Sá (UNESA/RJ). Mestre em Constitucionalismo e Democracia pela Faculdade de Direito do Sul de Minas (FDSM/MG). Pedagogo. Especialista em Neuropsicopedagogia. Pesquisador visitante no grupo de pesquisa em Direitos Humanos e Vulnerabilidade pela Universidade Católica de Santos - (UNISANTOS/SP) ORCID: https://orcid.org/ooooooo2-2908-0708 E-mail: tamiranda@yhaoo.com.
} 
immersed. Learning requires memory to carry out the operational and strategic processes in assimilation and knowledge management, through the interaction with the culture and environment. All of this, from an integrative posture, it highlights the importance of neuropsychopedagogy to obtain the necessary knowledgeabout the constructs or themes that originate and qualify the human learning.

Keywords: Neuropsychopedagogy - Memory - Learning

\section{INTRODUÇÃO}

aprendizagem pode ser definida como modificação estrutural e funcional dos sistemas neurológicos e biológicos, que permite apreender conhecimentos, emoções e condutas que, somadas às experiências, promovem o desenvolvimento.

Depende de diversos componentes e fatores genéticos, biológicos, ambientais, atitudinais, emocionais, bem como de elementos que são conformados no contexto em que a aprendizagem se apresenta. Isso significa que as experiências cotidianas, os meios educativos, as estratégias pedagógicas, o acompanhamento do aluno, entreoutros, são fatores que se referem à complexa trama de relações que devem ser estabelecidas para fortalecer as redes neuronais e as conexões sinápticas envolvidasna aprendizagem.

Para entender a extensão e a profundidade a aprendizagem do ser humano sepoderia retomar posturas que vão desde teorias condutistas e cognitivas até metodologias que envolvem a atividade mental superior, as quais situam a ação do sujeito cognoscente em um plano ecológico, porque na configuração da aprendizagemintervêm diversos elementos, como o meio externo, a estrutura subjetiva do sujeito, suas representações mentais, a forma como adquire os esquemas e seus níveis de processamento da informação.

Nesse ponto, a conduta, entendida não apenas como os atos que são observáveis externamente, mas também como produto da cognição e das emoções éo que se denomina aprendizagem ao longo da vida e um estado permanente de mobilidade cognitiva e emocional que ele assume para agregar à conduta novas formas de agir e processar informações.

Sendo assim, a aprendizagem não pode ser reduzida à explicação biológica, ou seja, a simples mudanças físico-químicas, à atividade neuronal, a fatores genéticosou aos aportes das ciências sociais.

Por esta razão, compreendendo a aprendizagem não como um elementoisolado 
ou um produto do súbito surgimento de algo que pode ser explicado apenas por um determinado campo, justifica-se a abordagem do tema da atuação neuropsicológica no desenvolvimento da aprendizagem, evocando como problema depesquisa a indagação: Qual a importância da atuação da neuropsicopedagogia para o desenvolvimento do processo de aprendizagem?

Tendo como foco o desenvolvimento dos sistemas de memória, compreende- se, ainda, que a abordagem dos fenômenos da aprendizagem requer uma visão intrinsecamente multidisciplinar e complexa, já que a multidisciplinariedade é uma dasvias para aumentar a qualidade do processo educativo e de formação integral, uma forma de compreender a realidade a partir do nexo entre diferentes disciplinas, promovendo e motivando formas de pensar que apontem a um objeto de estudo em comum.

Ao mesmo tempo, compreende-se que a neuropsicopedagogia fortalece a complexidade da realidade, integrando diversas neurociências condutais e não condutais para explicar os fenômenos do sistema nervoso central, como a memória, e suas correlações com o meio externo, cognitivo e emocional do indivíduo.

Com base nessa exposição, o objetivo geral do estudo é analisar a importânciada atuação da neuropsicopedagogia para o desenvolvimento do processo de aprendizagem, com foco no desenvolvimento dos sistemas de memória.

Seus objetivos específicos são: definir e caracterizar o processo deaprendizagem considerando o caráter multifatorial de seu conceito; revisar a relação entre a aprendizagem, o funcionamento executivo e a memória como complemento das funções executivas e destacar as contribuições da neuropsicopedagogia para a efetivação do processo de aprendizagem.

A metodologia do estudo é a pesquisa bibliográfica em diversos meios e fontes,tais como livros, revistas, periódicos e sites sendo, portanto, exploratória quanto às fontes de pesquisa e qualitativa quanto à análise dos dados coletados e discutidos emsua realização.

\section{NEUROPSICOLOGIA, APRENDIZAGEM E MEMÓRIA}

\section{I O caráter multifatorial do processo de aprendizagem}

Diversos estudos sobre a aprendizagem demonstram que esta é um processo multifatorial no qual incide um conjunto de variáveis que a facilitam ou dificultam, dentre 
as quais fatores cognitivos, afetivos, motivacionais, emocionais, interesses, necessidades, fatores sociais e/ou individuais do aluno, que incidem direta ou indiretamente na assimilação, compreensão e produção do conhecimento.

Wolfe (2014) comenta, nesse sentido, que os avanços nas produções cientificas revelam como a motivação e o ambiente influem no aluno quanto às suas interações, comportamentos e rendimento acadêmico, como os métodos ativos e o clima assertivo desenvolve a empatia e estimulam o aluno a querer aprender, como os estímulos afetivos desenvolvem emoções positiva que incidem de forma notável na qualidade da aprendizagem.

Essas perspectivas revelam, especialmente a partir do campo da neuroeducação, que na aprendizagem intervêm os fatores cognitivos, o interesse, a motivação, a satisfação e a alegria, as emoções positivas como condicionantes decisivos para o alcance de uma aprendizagem consciente e significativa.

O binômio emoção-cognição é indissolúvel, intrínseco à configuraçãoanatômica e funcional do cérebro e, quando há uma integração harmônica entre fatores cognitivos, afetivos e emocionais se torna possível desenvolver uma aprendizagem metacognitiva e autorregulada nos alunos. (WOLFE, 2014).

Também segundo Fonseca (2015), o processo de aprendizagem é tanto uma experiência intelectual, social, individual, como emocional, englobando a personalidade como um todo, potencializando as capacidades, os conhecimentos, as habilidades, a inteligência e as atitudes a partir de um enriquecimento integral que estimula a formação de sentimentos, valores, convicções, ideais e vivências significativas e permanentes nos alunos.

O enfoque neuropsicopedagógico da aprendizagem implica em considerar uma intervenção dos processos cognoscitivos, neuropsicológicos e psicopedagógicos,sem afastar os aspectos emocionais, cognitivos, neurológicos e condutais dessa análise. Como analisa Beauclair (2014), a aprendizagem é um tema desenvolvido primordialmente na área da educação e as neurociências tenham analisado osmecanismos anatômicos cerebrais dos processos de leitura, escrita e conhecimentosmatemáticos buscando encontrar estratégias para enfrentar as dificuldades dos alunos nesses processos.

Ainda, Beauclair (2014) assinala que a neurologia descreve a aprendizagem apartir da capacidade de adaptação do cérebro sob o princípio da plasticidade cerebral que, 
diferentemente da neurogênese, da apoteose ou da sinaptogênese, se refere à adaptação nas vias nervosas e nas sinapses, devido a mudanças de comportamentodos indivíduos como resposta ao meio, bem como a capacidade do cérebro para adaptar-se no caso de lesões físicas.

Isso evidencia como o orgânico é fundamental quando se aprende, pois se transforma o comportamento devido a algum estímulo e, indubitavelmente, o cérebrosofre mudanças quando esses processos ocorrem. Indo além, a compreensão a aprendizagem comporta também a ideia da aprendizagem mediada, na qual se entende que os estímulos do ambiente, transformados por um agente mediador, promovem a aprendizagem. (BEAUCLAIR, 2014).

Para Beauclair (2014), esse mediador, orientado por suas interações, sua cultura e sua carga emocional, seleciona e organiza os estímulos que rodeiam o aprendiz: os enquadra, filtra e etiqueta. $\mathrm{O}$ aprendiz adquire, assim, padrões deconduta e estratégias de aprendizagem que se convertem em ingredientes importantes em sua capacidade de ser modificado pela exposição direta aos estímulos.

Considerando estes diferentes pontos de vista, Beauclair (2014) ensina que é importante compreender a aprendizagem de forma multifatorial, na qual os aspectos que influem no indivíduo são considerados, permitindo um olhar integrador, proposto pela neuropsicopedagogia, que questiona como se fundamenta a concepção de aprendizagem.

Relvas (2016) discorre sobre o enfoque que enfatiza a aprendizagem humanacomo um reflexo de seu meio sócio-histórico (aprendizagem externa, sensório-motora e verbal), abordando a definição de aprendizagem como função criadora daquilo quese aprende e seus determinantes (a aprendizagem externa e interna, sensório-motorae verbal) e, por sua vez, a interrelação hierárquica que existe nos diferentes níveis deaprendizagem.

Somado ao anterior, para Relvas (2016), existe a necessidade de analisar o tema da evolução do processo de aprendizagem em relação com a capacidade dos organismos para adaptar-se ao meio, partindo da necessidade de sobrevivência das espécies e das mudanças que, em nível psicológico, estão contidos no processo. Portudo isso, a educação baseada no cérebro busca maximizar a aprendizagem, a memória, a atenção e o entendimento a partir de descobertas neurocientíficas e da evolução do cérebro.

Haase (2016) complementa esta afirmativa propondo um olhar multifatorial da aprendizagem mencionando que a condição mutável da aprendizagem faz com que oconceito de educação baseada no cérebro adquira uma dimensão mais ampla. É porisso que para esse 
processo, sob uma concepção holística, são considerados múltiplos fatores intervenientes. $\mathrm{O}$ processo educativo deve centrar-se no aluno, ser dirigido ao desenvolvimento da inteligência, desenvolvendo a capacidade de integração dos múltiplos processos que ocorrem nas dimensões cognitivas, afetivas emotoras, bem como também ao desenvolvimento de valores e capacidades individuais.

Também Relvas (2012) comenta que a neuropsicopedagogia infantil aborda diferentes pontos de vista teóricos para compreender os processos de aprendizagem.Especificamente, as neurociências contribuíram para responder a pergunta sobre como se aprende, interessando aos profissionais da educação e de outras disciplinascientíficas para gerar temas importantes de estudo, já que no cérebro se produzem mudanças quando se aprende.

Considerando as perspectivas teóricas que buscam responder a esse questionamento, o conceito de neuropsicopedagogia de Relvas (2012) se refere a esta como um exercício, um trabalho interdisciplinar sobre o processamento da informação e a modularidade da mente em termos de neurociência cognitiva, psicologia, pedagogia e educação, realizado por um profissional de formação multi e interdisciplinar e com fins educacionais.

Isso implica reconhecer o conceito atual da aprendizagem para realizar um exercício neuropsicopedagógico que aborde as necessidades a partir de um olhar multifatorial. (RELVAS, 2012).

Em complemento, Haase (2016) observa que a pedagogia aumentou seus interesses pelos estudos sobre o cérebro, partindo de uma análise de todos os fatoresque influem nas aprendizagens, contemplando a individualidade e necessidades do sujeito que aprende, sendo totalmente válido retomar a ideia de que é tarefa da neuropsicopedagogia desenvolver uma teoria heurística na qual podem se inserir, deforma criativa, os resultados obtidos na área das neurociências e da neuropsicologia, considerando que ambas as áreas buscam compreender o ser humano a partir de diferentes perspectivas.

\subsection{A relação entre a aprendizagem, o funcionamento executivo e a memória}

Os estudos sobre o cérebro desenvolvidos nos últimos anos o situam como o órgão que permite a diferenciação entre seres racionais e irracionais, tendo como umadas funções mais aperfeiçoadas a função executiva.

O conceito de função executiva foi aperfeiçoado por Luria (1986, apud BODROVA 
et al., 20II), que a definiu como uma atividade cognitiva, reguladora do comportamento humano, que permite efetuar atos delimitados por uma intenção definida para a obtenção de uma meta, favorecida pela mediação da linguagem.

Bodrova et al. (20II) também observam que foi Lezak quem ampliou esse conceito, afirmando que a função executiva é um conjunto de habilidades de planejamento, programação, regulação e verificação da conduta intencional. As funções executivas são capacidades que permitem a uma pessoa envolver-se com êxito em comportamentos independentes, com um propósito e uma autossatisfação definidas. Neste sentido, a função executiva tem um componente volitivo, de planejamento, comportamento proposital e desempenho efetivo que permite uma conduta socialmente responsável.

Por seu turno Stuss (1992, apud Bodrova et al., 20II), com o modelo hierárquico do controle executivo, define que a atividade pré-frontal se organiza segundo três níveis de processamento que são independentes no manejo da informação e se autorregulam interativamente através dos inputs e outputs que se geram entre eles. Estes três níveis são: processador de entrada da informação (nível perceptivo e sensorial automático); sistema comparador (antecipação, seleção de objetivos e elaboração de planos) que analisa a informação com base em experiências anteriores; sistema organizador de saída (autoconsciência e autorreflexão, que utiliza a informação do sistema comparador para escolher o tipo adequado de resposta).

Observam também Bodrova et al. (2011) que Burgess, em 1997 e Anderson, em 1998, descreveram a função executiva como um sistema multidimensional da conduta, do funcionamento emocional e da cognição humana. Esta definição indica que a função executiva envolve um aspecto amplo de funções corticais como a conduta dirigida a metas, a flexibilidade condutal e cognitiva, o controle do esforço, apreocupação, a organização, o planejamento do comportamento, a interferênciasocial, a inovação, a execução da ação e a linguagem.

Diante dessas abordagens, a contribuição de Bodrova et al. (2011) considera que as funções executivas representam a capacidade de orquestrar as funções cognitivas com habilidades de base, como a memória operativa, o controle inibitório eo monitoramento. Assim, como consenso entre os modelos que buscam aprofundar oestudo das funções executivas, admitem que são sistemas de operações cognitivas complexas, relacionadas com 
o funcionamento dos circuitos frontais, que se estruturam através de etapas sucessivas durante o desenvolvimento da linguagem eda inteligência geral.

Por outro lado, Diaz-Fuentes (2014) observa que são componentes das funções executivas as habilidades relacionadas com o controle inibitório, a memória operativa, as mudanças atencionais, os processos top-down e bottom-up ${ }^{2}$, a organização de processos seriais, a geratividade e a fluidez. Relacionando-se esses componentes com as diferentes dimensões das funções executivas, não está ainda claramente estabelecida como é a dinâmica dessa atividade cognitiva complexa; ou seja, se são mediadas pelas funções executivas ou em que medida essa mediação se relaciona com a estrutura do pensamento, o estilo cognitivo, a aprendizagem e o desenvolvimento das habilidades discursivas da linguaPara Diaz-Fuentes (2014), as funções executivas têm papel essencial nas condições que são necessárias para a aprendizagem, especialmente explicar os componentes cognitivos, condutais e emocionais inseridos nesta. Porém, a memória também é uma das funções cognitivasprincipais para retenção do conhecimento ou da informação, sendo, por isso, essencial para a aprendizagem como modificação de conduta.

Vários teóricos se referem a diversos tipos de memória para explicar a complexidade dessa função. Lee e Fong (2009) consideram que a memória é um processo cognitivo multissistêmico que permite a organização, armazenamento e evocação da informação.

Lee e Fong (2009) abordam em seu estudo a perspectiva de Tulving, desenvolvida em 1985 com relação ao funcionamento da memória, dividido em três grandes sistemas que trabalham conjuntamente: memória procedimental, semântica e episódica. A primeira memória procedimental - pode funcionar de forma isolada, sendo mais elementar e primitiva e que se encontra em todos os organismos, mesmo os mais simples. A memória procedimental compreende o conhecimento, em relaçãocom o saber como fazer as coisas e, segundo os autores, seria uma forma um pouco mais complexa de aprendizagem, diferentemente da conduta instintiva porque não requer a associação estímulo-resposta.

A memória semântica é a genérica ou de fatos particulares e não envolve nenhuma estrutura de eventos ou localização espaço-temporal. A memória episódicase refere a eventos

\footnotetext{
${ }^{2}$ Métodos utilizados para organizar as informações, ordenando fatos para serem utilizados emdiversos campos que necessitem de uma forma de pensamento que facilite a administração deconhecimentos para um maior aproveitando destes, com maior facilidade de processamento e garantindo um sistema de prioridades e hierarquia. (ENSINO: GUIA DE EDUCAÇÃO, 2020).
} 
que ocorreram em um local e tempo específicos e a recuperaçãodesse tipo de informação geralmente requer a criação de imagens mentais ou mapasmentais que são evocados no momento e cuja recordação pode incluir grande variedade de tipos de informação (visual, auditiva, olfativa, temporal, espacial, emotiva, etc.). Este processo pode recriar o evento passado de forma viva nopresente. (LEE; FONG, 2009).

Mencionam Corso et al. (2013) a sincronia entre a função executiva e amemória gera uma articulação coerente com o desenvolvimento das diversas áreas do córtex préfrontal, citoarquitetura onde se localizam as funções executivas e a memória em suas dimensões temporais, contextuais, práticas e emocionais. Tudo issoé necessário para gerar transformações na conduta, ou seja, aprendizagens influenciadas pela relação do sujeito com o meio externo e suas capacidades intrínsecas, como o processamento da informação.

É importante precisar que a psicologia cognitiva realizou diversas investigações relacionadas com a compreensão da mente humana e alguns estudos, como advertem Corso et al. (2013), se propuseram a estabelecer uma taxonomia da memória, explicar seu funcionamento e, fundamentalmente, a incidência desse processo cognitivo no armazenamento, retenção e manejo da informação, como algumas formas que permitem a aquisição de aprendizagens que não são, estritamente, as aprendizagens escolares.

Sem a retenção e o manejo da informação não seria possível questionar, processar e assimilar tudo aquilo que vem do ambiente em que o indivíduo se encontraimerso e por isso, segundo Corso et al. (2013), é importante a intersecção desubsistemas psíquicos superiores, como a memória e as funções executivas para explicar a maneira como se aprende e como é a adaptação do indivíduo quando desenvolve aprendizagens em situações novas.

Conforme Lee e Fong (2009), a memória se estende como uma macroestruturaque conta com um sistema complexo e ordenado e subsistemas cerebrais divisíveis que interagem e interatuam permanentemente com outros processos cerebrais que se expressam em condutas, emoções, a cognição e a experiência. A memória se desenvolve em diferentes momentos e se classifica em três níveis, de acordo com o momento em que se encontra (imediata, mediata e de longo prazo). A primeira se refere a toda informação que chega ao cérebro através dos sentidos, não permanecendo por longos períodos de tempo, dependendo do seu impacto - se a informação não é importante, é descartada.

Complementam Lee e Fong (2009) que quando os sinais recebidos são importantes e 
despertam interesse o estímulo externo (sensorial) ou interno (sensações, emoções, pensamentos) dão origem a uma nova instância, que alcançaoutro nível de memória. A memória mediata, memória de trabalho ou funcional, portanto, é aquela que guarda sinais que vêm dos sentidos por curtos períodos de tempo e atua sobre eles.

Lee e Fong explicam essa dinâmica:

A informação percebida é transferida para a memória de trabalho, na qual serecorda, aproximadamente, o que se percebe. A particularidade desta memória é que guarda e ao mesmo tempo processa os sinais recebidos e outras memórias. A memória de trabalho é um mecanismo de armazenamento temporário da informação, que favorece a retenção dealguns dados na mente para estabelecer relações entre eles ou compará-losno caso de processos cognitivos de alta complexidade, entre os quais se encontra a aprendizagem. (LEE; FONG 2009, p. 379).

A memória de longo prazo se refere a tudo o que foi aprendido ou visto, que se armazena de forma verbal e visual, de forma independente, mas interconectada, ou seja, toda a informação de menor complexidade que uma pessoa pode reter (datas, nomes, etc.) e outras mais complexas, como a formulação de novas hipóteses para resolução de problemas. Estas informações se encontram inativa no cérebro, alerta diante de qualquer demanda do ambiente ou tarefa a realizar. (LEE; FONG, 2009).

A importância de cada uma destas no âmbito da memória de trabalho é fundamental aos processos de aprendizagem, entendidos como aqueles por meio dosquais se adquirem novos conhecimentos. A memória seria a base primordial para reter esse conhecimento. Durante a consolidação, as novas recordações não se conservamaleatoriamente no cérebro, mas têm uma relação direta com outras recordações do mesmo tipo. Toda a informação que o cérebro guarda se gera através de mudanças na comunicação sináptica, que é o processo mediante o qual um neurônio secomunica com outro. (HAASE, 2016).

Por outro lado, Haase (2016) observa que nos estilos de aprendizagem se encontram envolvidos tipos de memória que respondem a diferentes sinais, tarefas ouatividades a serem desempenhadas. Para aprender alguma atividade que requeira motricidade (realizar um cálculo ou aprender música, uma nova lição, a compreensãode um texto, etc.), estão envolvidos diferentes sistemas de memória.

De acordo com isso, existe a possibilidade, segundo Haase (2016), de encontrar pessoas que contam com excelentes habilidades para reter e guardar informações, mas não necessariamente sua compreensão, pois a memória, neste caso, se encarrega de guardar palavras, mas não ideias que permitam codificar a informação abstraída. Por isso, é possível 
que uma pessoa lembre o tema sobre o qual se fala, tenha uma vaga ideia, mas não possua a habilidade cognitiva de coordenar e organizar a informação para compreender e interiorizar a ideia com profundidade. Essa questão remete às memórias de curto e longo prazo necessáriaspara exercer as funções básicas da aprendizagem, reconhecendo-se que nem todos os alunos contam com as mesmas habilidades.

A recuperação de informações armazenadas em outro momento seria a evidência de uma nova aprendizagem, que passou por todos os processos defuncionamento da memória no indivíduo e se instaurou de maneira eficaz. Desse modo, as funções executivas e a memória, embora tenham períodos de desenvolvimento, são processos que se potencializam, desenvolvem e harmonizam ao longo da vida. Por isso é imprescindível, dentro dos processos formativos, atribuir-lhes importância. Ainda que seja o aluno responsável por armazenar suas lembrançase informações, o professor e as pessoas envolvidas em sua formação têm um papel fundamental na potencialização da mesma para fortalecer sua aprendizagem, regular sua conduta e provocar recordações mais duradouras. (HAASE, 2016).

Esse aspecto distintivo da aprendizagem é citado também por Beauclair (2014), quando

reconhece que o determinante na recordação ou lembrança de dados e informações é o nível de processamento, muito mais do que a intenção de recordar.

Ao mesmo tempo, Beauclair (2014) assinala que a aprendizagem se realiza espontânea ou intencionalmente. A aprendizagem espontânea está explícita emtendências inatas ou pelo número de experiências repetitivas que levem a associações, como é o caso da linguagem. A aprendizagem intencional requerintervenções pedagógicas nas quais participem professores, pais, psicólogos e demais agentes pedagógicos que podem reconhecer a maneira como o indivíduo aprende, de acordo com sua capacidade para reter informações, graças à repetição que lhe permita recordá-las e codificar a informação através de situações novas em que sinta a necessidade de evocá-la, a partir da elaboração de relações que levem a um novo conhecimento.

\subsection{Contribuições da neuropsicopedagogia para a efetivação do processo deaprendizagem em sala de aula}

As contribuições da neuropsicopedagogia quanto à memória e a aprendizagemsão importantes para a compreensão de que as funções executivas adotam diversase múltiplas 
considerações, a partir de posições acadêmicas diversas, mas que têm um ponto comum: consideram que as funções executivas são sistemas de operações cognitivas complexas, relacionadas com o lóbulo frontal, onde se estruturam o desenvolvimento da linguagem e o conhecimento generalizado. (RELVAS, 2016).

Para Relvas (2016), o aprofundamento nas teorias neuropsicopedagógicas leva à compreensão de que as funções executivas e a memória, como processos psicológicos superiores, se vinculam e interatuam para facilitar a aprendizagem, conforme a maneira como os indivíduos retêm a informação e a utilizam para gerar respostas adaptativas aos sistemas externos em que participam.

Gerenciar corretamente o conhecimento destas teorias permite, segundo Relvas (2016), compreender que a modificação estrutural atribuída pela aprendizagem gera novos estilos cognitivos, fortalece a atividade mental superior, participa no controle e regula a conduta dos aspectos emocionais.

Como são requeridos processo operativos e estratégicos para consolidar a aprendizagem, como forma de reter, associar e renomear a informação obtida através da memória, o domínio desses conhecimentos permite desenvolver experiências quefavorecem a aprendizagem e a reforçam por toda a vida. (RELVAS, 20I6).

Wolfe (2014) ressalta essa importância ao afirmar que os estudos em neuropsicopedagogia levaram à constatação de que a memória possui três funções principais para a construção da aprendizagem: retêm e guarda as informaçõesrecebidas pelos sentidos; organiza e codifica em representações mentais essas informações, para que possuam um sentido, o qual depende em grande medida da atenção para selecionar o que verdadeiramente é significativo; recupera o que é necessário para a tarefa no qual seja exigido ou em algum momento no qual deva serrecordado um elemento específico. Esta é a forma pela qual os seres humanos acedem ao banco de dados armazenado em seu cérebro e que pode ocorrer de formaespontânea ou voluntária.

Nesse sentido, os processos de aprendizagem requerem conhecimentos paraauxiliar o indivíduo a gerar uma conexão com um ambiente estimulante que lhe ofereça a possibilidade de evocar recordações, um ambiente que proporcione novas conexões sinápticas ou formação de redes neuronais para a construção de associações entre a informação de entrada e de saída, que se armazenem essas informações na memória de longo 
prazo para que em outro momento possamconsolidar novos conhecimentos. (WOLFE, 2014).

Do ponto de vista neuropsicopedagógico, para Wolfe (2014, p. 72), "os lóbulosfrontais representam um sistema de planejamento, regulação e controle dos processos psicológicos”. Devido ao envolvimento destes na capacidade do serhumano para planejar e regular a conduta e os processos psicológicos superiores,estes são o centro das funções executivas e o processo educacional não podedesconhecer essa informação para favorecer aprendizagens significativas e exitosas. No mesmo sentido, para Wolfe (2014), a função executiva, dada a sua natureza autorreguladora, antecipatória, previsora de metas, de projeção de programas,incitadora de atividades e de operações mentais, monitora de tarefas, seletora decomportamentos, organizadora e flexibilizadora no cérebro humano, facilita o ensinoe a aprendizagem em termos de desenvolvimento dos processos cognitivos superiores.

Dito de outro modo, no ambiente educativo as funções executivas e a memóriaintervêm em uma série de cenários de aprendizagem e seu desenvolvimento deve serregra em todos os contextos educacionais.

Por fim, como observa Wolfe (2014), as contribuições da neuropsicopedagogiapara a efetivação do processo de aprendizagem são visíveis em termos de qualificação do processo ensino-aprendizagem. As posturas científicas neuropsicológicas, psicológicas e pedagógicas com relação à tríade funções executivas-memória-aprendizagem demonstram o papel fundamental dos lóbulosfrontais, mas, ao mesmo tempo, reconhecem a multifatorialidade que envolve todo esse processo de construção de referências vitais, guiadas por contingências e atos voluntários como é a aprendizagem.

Da mesma forma, Wolfe (2014) afirma que a memória e as funções executivas, corretamente compreendidas, permitem a evolução no desenvolvimento dosindivíduos, permeando-os de novas formas para conceberem o real. Esta é outra forma de explicar as contribuições da neuropsicopedagogia para a aprendizagem, a partir dos fatores que se integram para a compreensão de como o ser humano aprende e como utiliza essa aprendizagem para adaptar-se e continuar evoluindo.

\section{CONSIDERAÇÕES FINAIS}


Este estudo abordou de maneira reflexiva uma análise pedagógica da atuação da neuropsicopedagogia para o desenvolvimento da aprendizagem e da memória e cumpriu com o objetivo de analisar a importância da atuação da neuropsicopedagogia para o desenvolvimento do processo de aprendizagem, com foco no desenvolvimentodos sistemas de memória.

Inicialmente, abordou o caráter multifatorial do processo de aprendizagem, concluindo que a essência da aprendizagem consiste no surgimento e modificação dos processos psíquicos e do comportamento tanto em um aspecto ou dimensão afetiva como no aspecto cognitivo. Aprende-se não somente hábitos, conhecimentos, habilidades e capacidades, mas também atitudes, aspectos volitivos, emoções, sentimentos e necessidades e, por isso, a aprendizagem se refere tanto ao processo de ensino como ao processo de educação.

Tratou também da relação entre a aprendizagem, o funcionamento executivo ea memória. Dessa abordagem conclui que as funções executivas e a memória estão interconectadas por diversos fatores que demonstram o funcionamento complexo da atividade cerebral, presente nos sistemas neuronais, cujas funções estabelecem a consciência, a iniciação e o monitoramento de atividades exigidas pelo ambiente, as decisões, o controle, a linguagem, o significado das informações, a memória e os procedimentos e atos eu estabelecem uma base para o desenvolvimento e a manutenção da aprendizagem do ser humano.

Desta abordagem deriva o terceiro foco do estudo, que são as contribuições da neuropsicopedagogia para a efetivação do processo de aprendizagem. Nesse sentido compreendeu-se, conclusivamente, que a neuropsicopedagogia se preocupa ementender como o indivíduo aprende e intervir, potencializando habilidades e reconhecendo as necessidades - nem sempre orgânicas - de aprendizagem.

Assim, é essencial que as teorias neuropsicopedagógicas sejam aplicadas à aprendizagem em um enfoque multidimensional e não fragmentado para unificar e integrar os diversos aspectos que intervêm para que um indivíduo aprenda ou não de maneira adequada, tendo em consideração também os fatores externos que podem afetar tanto o indivíduo como os ambientes em que ele interage.

Finalmente, conclui-se que aprendizagem, o funcionamento executivo ou as funções 
executivas e a memória, processos psicológicos de ordem superior, não sãoisolados. Pelo contrário, a memória é o complemento das funções executivas e ambospermitem ao indivíduo gerar respostas adaptativas aos sistemas nos quais se encontra imerso.

A aprendizagem necessita da memória para realizar os processos operativos e estratégicos na assimilação e na gestão do conhecimento, através da interação com a cultura e o meio. Tudo isso, a partir de uma postura integradora, destaca aimportância da neuropsicopedagogia para obter o conhecimento necessário sobre osconstrutos ou temáticas que dão origem e qualificam a aprendizagem humana.

\section{REFERÊNCIAS}

BEAUCLAIR, João. Neuropsicopedagogia: inserções no presente, utopias e desejos futuros. Rio de Janeiro: Essence All, 2014.

BODROVA, Elena LEONG, Deborah J.; AKHUTINA, Tatiana V. When everything new is wellforgotten old: Vygotsky/Luria insights in the development of executive functions. New Directions for Child and Adolescent Development, (I33), II-28, 2011.

CORSO, Helena Vellinho; SPERB, Tânia Mara; DE JOU, Graciela Inchausti; SALLES, Jerusa Fumagalli. Metacognição e funções executivas. relações entre os conceitos e implicações para a aprendizagem. Disponível em: <www.scielo.br/pdf/ptp/v29n5/o4.pdf >. Acesso em 5 mar. 2020.

DIAZ-FUENTES, Daniel. Neuropsicologia: teoria e prática. 2. ed. Porto Alegre: Artmed, 2014 .

FONSECA, Vitor. Cognição, Neuropsicologia e Aprendizagem. Petrópolis, Vozes, 2015.

HAASE, Vitor Gerald. Aprendizagem e neuroplasticidade. In: METRING, Roberte; SAMPAIO, Simaia (org.). Neuropsicopedagogia e Aprendizagem. Rio de Janeiro: WAK Editora, 20I6, p. 8I-88.

LEE, Kerry; NG, Eelynn; FONG, Swee. The contributions of working memory and executive functioning to problem representation and solution generation in algebraic word problems. Journal of Educational Psychology, n. Ior, a. 2, p. 373-387, 2009.

RELVAS, Marta Pires. Neurociência do aprendizado e a sala de aula. In: METRING, Roberte; SAMPAIO, Simaia (org.). Neuropsicopedagogia e aprendizagem. Rio de Janeiro: Wak Editora, 2016, p. 199-205.

RELVAS, Marta. Neurociência na prática pedagógica. Rio de janeiro: Wak Editora, 2012.

Você sabe o que são os processos de tot-down e bottom-up? Ensino: guia de educação. 
Disponível em: https://canaldoensino.com.br/blog/voce-sabe-o-que-sao-os-processos-detop-down-e-bottom-up. Acesso em 5 mar. 2020.

WOLFE, Patrícia. Compreender o funcionamento do cérebro e a sua importância paraa aprendizagem. 3. ed. Porto: Porto Editora, 2014. 